\title{
A reply to a commentary on identification of the rare compound heterozygous variants in the NEB gene in a Korean family with intellectual disability, epilepsy and early-childhood-onset generalized muscle weakness
}

Journal of Human Genetics (2015) 60, 163-164; doi:10.1038/jhg.2014.119; published online 15 January 2015

We thank Pelin et al. ${ }^{1}$ for their commentary on our article. ${ }^{2}$

As a result of the quintet-based wholeexome sequencing analysis, a total of seven compound heterozygous variant pairs of five genes were identified as candidate variants corresponding to the autosomal recessive genetic model (Supplementary Table S1). Next, we determined the allele frequency of the candidate variants in an Asian population on the basis of the data from the 1000 Genomes Project (http://www.1000genomes. org/), and selected those with an allele frequency of $1 \%$ or less. Finally, only one compound heterozygous $N E B$ variant pair, which is unique in the two affected siblings, was found.

The two affected siblings had the same compound heterozygous variation in the $N E B$ gene encoding nebulin, which was composed of two different missense variants: c.2603T $>$ C (p.L868P) in exon 27 and c.21340C $>$ T (p.R7114W) in exon 143. The allele frequency in the Asian population from the 1000 Genomes Project is 0.010 for c.2603T4C and 0.003 for c.21340C4T. Therefore, there is a $0.003 \%$ chance $(0.010 \times 0.003 \times 100)$ that it will be the compound heterozygous variation composing both c.2603T $>\mathrm{C}$ and c.21340C $>\mathrm{T}$ in a subject. On the basis of the extremely low allele frequency, in silico prediction and literature review, as well as the myopathic findings on the needle electromyography study, we concluded that this compound heterozygous $N E B$ variation may be a sound candidate for the disease-causing mutation in this family. The mutation update on the NEB that you mentioned did not have the same compound heterozygous variation that our two affected siblings have. ${ }^{3}$ The p.R7114W variant is not included in Exome Variant Server or your recent mutation update on the $N E B$, either. Exon 143 is involved in developmental stage-specific alternative splicing and might harbor a regulatory function utilized during muscle maturation. ${ }^{4}$ You mentioned that the intellectual disability, epilepsy or any other brain abnormality were not present in any of the patients in your study. Although the function of nebulin in the brain is unknown, nebulin is one of actin regulators. Cytoskeletons such as actin and microtubule have critical roles in synaptic functions of the brain. ${ }^{5-8}$ Formation of synapses and synaptic plasticity requires cytoskeletons. ${ }^{5-8}$ Abnormalities of actin-binding proteins could cause dysfunction of the actin cytoskeleton. As nebulin is an actin regulator like dystrophin, ${ }^{9,10}$ it seems that synaptic excitability and synaptic plasticity could be altered by a dysfunction of actin cytoskeleton caused by $N E B$ mutations.

Several study limitations should be noted in our research, as we mentioned. Because the affected family is quite small and there are no strong linkage data in this family, more complex non-monogenetic mechanisms involved in the identified $N E B$ variations need to be considered. In addition, functional studies are required to elucidate the possible mechanisms underlying the central nervous system dysfunction in these rare compound heterozygous $N E B$ variants.

We agree with the critique by Pelin et al. on technical limitations. However, we believe that this compound heterozygous NEB variation as a potential diseasecausing mutation warrants further studies because of the intriguing and novel combination of severe muscle weakness with intellectual disability and epilepsy in this family.

Shin-Young Yim ${ }^{1,2}$ and Seon-Yong Jeong ${ }^{1}$

${ }^{1}$ Department of Medical Genetics, Ajou University School of Medicine, Suwon, Republic of Korea and ${ }^{2}$ Department of Physical Medicine and Rehabilitation, Ajou University School of Medicine, Suwon, Republic of Korea E-mail:syyim@ajou.ac.kr.

\footnotetext{
1 Pelin K., Kiiski K., Lehtokari V. L. \& Wallgren-Pettersson C. A commentary on identification of the rare compound heterozygous variants in the $N E B$ gene in a Korean family with intellectual disability, epilepsy and early-childhood-onset generalized muscle weakness. J. Hum. Genet. 60, 161-162 (2015)

2 Jin H. S., Lee J. B., Kim K., Lee K. Y., Choi V. N., $\mathrm{Kim}$ J. S. et al. Identification of the rare compound heterozygous variants in the $N E B$ gene in a Korean family with intellectual disability, epilepsy and earlychildhood-onset generalized muscle weakness. J. Hum. Genet. 59, 643-647 (2014).

3 Lehtokari, V. L., Kiiski, K., Sandaradura, S. A., Laporte, J., Repo, P., Frey, J. A. et al.
} 
Mutation update: the spectra of nebulin variants and associated myopathies. Hum. Mutat. 35, 1418-1426 (2014).

4 Donner, K., Nowak, K. J., Aro, M., Pelin, K. \& Wallgren-Pettersson, C. Developmental and muscle-type-specific expression of mouse nebulin exons 127 and 128. Genomics 88, 489-495 (2006)
5 Kneussel, M., Triller, A. \& Choquet, D. SnapShot: receptor dynamics at plastic synapses. Cell 157 1738-1738 e1731 (2014).

6 Cai, Q. \& Sheng, Z. H. Molecular motors and synaptic assembly. Neuroscientist 15, 78-89 (2009).

7 Hotulainen, P. \& Hoogenraad, C. C. Actin in dendritic spines: connecting dynamics to function. J. Cell Biol. 189, 619-629 (2010)
8 Conde, C. \& Caceres, A. Microtubule assembly, organization and dynamics in axons and dendrites. Nat. Rev. Neurosci. 10, 319-332 (2009).

9 Siripala, A. D. \& Welch, M. D. SnapShot: actin regulators I. Cell 128, 626 (2007).

10 Siripala, A. D. \& Welch, M. D. SnapShot: actin regulators II. Cell 128, 1014 (2007).

Supplementary Information accompanies the paper on Journal of Human Genetics website (http://www.nature.com/jhg) 